\title{
Is guideline-driven prophylaxis for venous thromboembolism common practice in the South African private hospital setting?
}

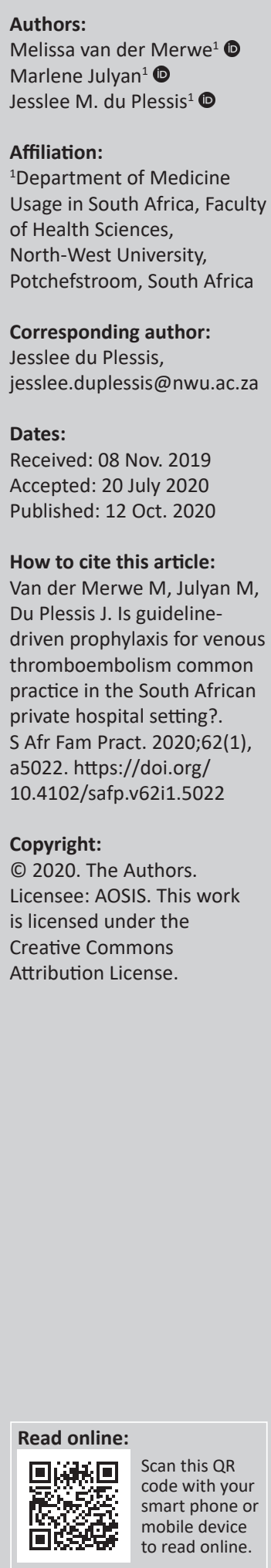

Background: Prophylactic venous thromboembolism (VTE) strategies have the greatest impact on patient outcomes. Both global and local guidelines support VTE prophylaxis for hospitalised patients. However, studies have reported that these measures are routinely under-prescribed. This study evaluated prescribing patterns of VTE prophylaxis in one of the largest South African (SA) private hospital groups.

Methods: A quantitative, retrospective analysis of the hospital group's patient database was conducted for patients admitted between 01 September 2015 and 31 August 2016. Those younger than 18 years with trauma or suffering from contraindications to anticoagulation were excluded. Additionally, patients with warfarin billed were also excluded as they possibly required therapeutic anticoagulation. Included prophylactic measures were compared with published SA guidelines by abstracting prophylaxis type and dosing, according to corresponding individual patients' VTE risk ratings.

Results: Amongst the 373020 patients included as the study population, $77 \%$ required prophylaxis. Of these, $38.36 \%(n=85486)$ received guideline-appropriate prophylactic measures during their hospital stay. Patients in whom prophylaxis was indicated, only $24.56 \%$ $(n=42715)$ complied with the SA guidelines. The most commonly used prophylactic measures were enoxaparin $(89.09 \%)$ and fondaparinux (2.68\%). Prophylactic measures differed per speciality, with the most compliant amongst intensivists. A low uptake of the risk assessment model use $(n=222860,59.75 \%)$ was, however, reported for this data set.

Conclusion: Less than $24.56 \%$ of patients who required prophylaxis received guidelineappropriate interventions. Further studies should focus on understanding differences in practice and improving acceptance and application of guideline-driven care.

Keywords: venous thromboembolism; prophylaxis; hospital; South Africa; risk assessment.

\section{Introduction}

The global incidence of venous thromboembolism (VTE) has been reported to be exceptionally high with an annual overall prevalence rate similar to that of stroke, the fifth leading cause of death worldwide. ${ }^{1}$ A multinational, observational, cross-sectional survey was carried out with 1583 patients from five sub-Saharan countries. A 50.4\% VTE prevalence rate was reported amongst at-risk patients from urban specialised hospitals. The majority $(62.3 \%)$ of those at risk were classified under a medical speciality, whilst surgical patients made up $43.8 \%$ of sufferers. ${ }^{2}$ For South Africa, the risk of VTE development during 2008 in hospitalised patients from parts of its Gauteng province was described to be at $74.6 \%$, with venous thromboembolic-related deaths reported at around 20000 persons annually in 2012.,34 A lack of updated data currently exists, and because most VTE symptoms remain undetected, the true VTE incidence together with its possible impact on the South African (SA) private healthcare system largely remains unknown.,56

Venous thromboembolic disease not only is debilitating but also presents a high economic burden on a country's healthcare system. This is mainly because of a $45 \%$ increased cost for recurrent hospitalisation with VTE-related comorbid diseases. ${ }^{7}$ Recurring VTE-related hospitalisations often require a $48 \%$ increased expenditure when compared with the initial admission period..$^{8}$ It is estimated that the highest cost is suffered during the first 3 days after re-hospitalisation, possibly because of the higher level of care required. ${ }^{9}$ Up to $24 \%$ of patients diagnosed with VTE will eventually require intensive care unit re-admission. ${ }^{9}$ In 2014, VTE-related hospitalisation cost the United States healthcare system around \$10 billion, and in 2017, it was established through personal communication that one of South Africa's largest private hospital groups spent over 
R195 million on VTE prophylactic and treatment measures. ${ }^{7}$ This may indicate VTE management being one of the most expensive medical strategies.

Venous thromboembolic development has traditionally been attributed to patients already hospitalised for extended periods of time. ${ }^{10}$ However, approximately $25 \%$ - $40 \%$ of non-hospitalised patients are at risk of VTE development. ${ }^{10}$ Pharmacological VTE prophylaxis is unfortunately not without hazard, as its underlying mechanism may result in life-threatening haemorrhage. ${ }^{11}$ It is therefore important to avoid pharmaceutical prophylaxis when the benefit does not outweigh the risk. ${ }^{12}$ For these reasons, patient risk stratification on admission is paramount in order to ensure that at-risk patients receive the correct type and dose of prophylaxis without incurring additional harm. ${ }^{12}$ It has been proven that supplementary to risk stratification, implementation of VTE prophylactic guidelines results in improved patient outcomes. ${ }^{13}$ Yet, VTE-related complications because of poor prophylactic practices have given rise to $64.4 \%$ of all premature deaths in high-income countries and $66 \%$ in low- and middleincome countries. ${ }^{14,15}$ This may be attributed to variances in prophylaxis used on patients. ${ }^{15,16,17,18}$ The SA arm of 'The Use of VTE prophylaxis in relatioN to patiEnt risk profiling' (TUNE-IN) study found that only $67.9 \%$ of patients rated as possessing a high risk for VTE development received appropriate prophylaxis. ${ }^{3}$ Interestingly, it is reported that the main reason for reduced prophylactic prescribing is prescribers' perception that patients have a decreased risk forVTE development compared to availableepidemiological data. ${ }^{3}$ A large discrepancy often exists in the perceived VTE development risk amongst patients clinically appraised versus those in whom a standardised risk assessment model (RAM) was completed. ${ }^{3}$ Risk assessment models are traditionally designed to select patients in whom VTE prophylaxis benefit would outweigh the risk. ${ }^{3}$ Several reviews exist on the different available RAMs' ability to predict VTE development; however, no consensus has been reached on a preferred model..$^{19,20}$ The American College of Chest Physicians' (ACCP) VTE prophylaxis guidelines are generally regarded as the gold standard to be followed, and Caprini devised a RAM to enable the easy implementation of these guidelines. ${ }^{13,21}$ The Caprini RAM is founded on a point-based scoring method where points are awarded to risk factors according to their propensity for VTE. ${ }^{13}$ Based on the total calculated per patient, a low, medium or high risk for VTE development is awarded..$^{13}$ The RAM by Caprini is also the only model that has been externally validated for its VTE prediction ability and can be used in medical as well as surgically ill patients. ${ }^{22,23,24} \mathrm{~A}$ modest reduction was furthermore detected in VTE prevalence after patients were screened using the Caprini RAM and its suggested prophylaxis initiated.22 This RAM possibly positions itself firmly as a tool to reduce VTE occurrence in patients, improve patient care and reduce medical-related expenses when used correctly.
Venous thromboembolism is considered one of the most expensive and common preventable causes of global mortality. Despite its implication, there exists a scantiness of data available to describe VTE risk and prophylactic measures across the private hospital sector in South Africa (which is required to reduce healthcare costs). Local, peerreviewed VTE prophylactic guidelines have been published by the South African Society of Thrombosis and Haemostasis (SASTH). ${ }^{25}$ These guidelines are based on those prescribed by the ACCP, are peer reviewed and are user-friendly in their application. This study aimed at describing the VTE risk and prophylactic practices in the private sector across a large area of South Africa, by comparing practices to those prescribed by SASTH guidelines. This may possibly lay the foundation for patient outcome improvement.

\section{Methods Study design}

A quantitative, retrospective data analysis was performed. The sample included all patient data of those admitted over a 1-year period as 'inpatients'. Patients admitted as day cases or outpatients were excluded from the analysis. Data were analysed by the Department of Statistics, Faculty of Health Sciences at North-West University in South Africa.

\section{Setting}

A private hospital group was selected as the study setting, as prescriber habits are not governed by healthcare sector formularies or restrictions. The study setting comprised 54 hospitals located in seven of the nine SA provinces (no representation for Limpopo and the Northern Cape and only one hospital for the North West province). Services rendered included general medicine, surgery (acute or specialised), obstetrics and gynaecology, internal medicine, cardiology and medical oncology as part of a tertiary hospital offering. Most admissions $(23.98 \%, n=106085)$ for the study period were for referrals and admissions under general practitioners, with $22.48 \%$ ( $n=94455)$ admissions for surgically related procedures and $14.22 \%(n=62$ 914) admissions for gynaecological or obstetric procedures.

\section{Data source}

All admission and coding information, according to the 10th revision of the International Statistical Classification of Diseases and Related Health Problems (ICD10), could be cross-linked to in-hospital pharmacy billing data. This enabled the abstraction of the type of prophylactic use with its dosing, frequency as well as duration. Prophylactic medicine data could then be cross-linked to the principal diagnosis and the VTE risk of the individual patient could be calculated by using unique patient admission numbers. Venous thromboembolism risk ratings were calculated by nursing staff during patient admission, as is the standard practice for the group. This was performed by utilising a modified version of the Caprini RAM with results 
captured by clinical case managers on patient admission profiles. Venous thromboembolism risk ratings were captured in 59.74\% $(n=222860)$ of the study participant data meeting the inclusion criteria. Risk ratings were absent for $40.25 \%(n=150160)$ of the patient data. As a result of the nature of the study, patients without VTE risk ratings' data had to be excluded from guideline comparison, as no inference could be made on the appropriateness of prophylactic use. A summary of each risk factor and score, as contained in the modified Caprini RAM, is presented in Table 1 and a diagrammatical representation of the study sample data handling is outlined in Figure 1.

The modified Caprini RAM differed from the originator by classifying obesity as having a body mass index (BMI) of 25, whereas Caprini's classification included a BMI of over 30. Further differences included the exclusion of creatinine clearance, factor V Leiden levels, prothrombin 20210A

TABLE 1: Risk assessment model from study setting.

\begin{tabular}{|c|c|}
\hline $\begin{array}{l}\text { Modified Caprini RAM risk } \\
\text { category }\end{array}$ & Patient characteristics \\
\hline $\begin{array}{l}\text { Low VTE development risk } \\
\text { (risk factors assigned } 1 \\
\text { point each) }\end{array}$ & $\begin{array}{l}\text { - Patients between } 41 \text { and } 60 \text { years of age } \\
\text { - Body mass index of }>25 \\
\text { - Patients currently suffering from swollen legs } \\
\text { - Varicose veins } \\
\text { - Medical patient currently at bed rest } \\
\text { - Planned minor surgery } \\
\text { - Myocardial infarction (acute) } \\
\text { - Abnormal pulmonary function/chronic obstructive } \\
\text { pulmonary disease } \\
\text { - History of inflammatory bowel disease } \\
\text { - History of prior major surgery in the last } 30 \text { days } \\
\text { - Suffering from congestive heart failure in the last } \\
30 \text { days } \\
\text { - Sepsis in the last } 30 \text { days } \\
\text { - Different lung diseases including pneumonia in the } \\
\text { last } 30 \text { days } \\
\text { - Women who are pregnant or postpartum } 30 \text { days } \\
\text { - Women who are taking oral contraceptives or } \\
\text { hormone replacement therapy } \\
\text { - Females with a history of unexplained stillborn } \\
\text { babies or having had more than three recurrent } \\
\text { spontaneous abortions, toxaemia resulting in } \\
\text { premature births or patients with an infant showing } \\
\text { slowed growth }\end{array}$ \\
\hline $\begin{array}{l}\text { Medium VTE development } \\
\text { risk (risk factors assigned } \\
2 \text { points each) }\end{array}$ & $\begin{array}{l}\text { - Age between } 61 \text { and } 74 \text { years } \\
\text { - Those with a central venous line } \\
\text { - Current or prior malignancy } \\
\text { - Immobilised patients with plaster cast in the last } \\
30 \text { days } \\
\text { - Patients undergoing arthroscopy } \\
\text { - Immobilised patients of } 72 \mathrm{~h} \text { and longer } \\
\text { - Planned surgery of longer than } 44 \mathrm{~min}\end{array}$ \\
\hline $\begin{array}{l}\text { High VTE development risk } \\
\text { (risk factors assigned } \\
3 \text { points each) }\end{array}$ & $\begin{array}{l}\text { - Patients older than } 75 \text { years } \\
\text { - Patient history of deep vein thrombosis and } \\
\text { pulmonary embolism } \\
\text { - Patient familial thrombosis history }\end{array}$ \\
\hline $\begin{array}{l}\text { High DVT development risk } \\
\text { (risk factors assigned } \\
5 \text { points each) }\end{array}$ & $\begin{array}{l}\text { - Those suffering multiple trauma in the last } 30 \text { days } \\
\text { - Patients suffering from paralysis or acute spinal cord } \\
\text { injuries during the last } 30 \text { days } \\
\text { - Those with pelvic or hip fractures during the last } \\
30 \text { days } \\
\text { - Patients with planned hip or knee orthoplastic } \\
\text { replacement }\end{array}$ \\
\hline
\end{tabular}

DVT, deep vein thrombosis; RAM, risk assessment model; VTE, venous thromboembolism. levels, serum homocysteine levels, anticardiolipin antibody as well as Lupus antibody tests in the modified RAM. The modified Caprini RAM remains useful and was verified for settings where it may be impractical to conduct tests such as Lupus antibody assays because of time or financial constraints. ${ }^{26}$

\section{Data collection}

Data consisted of all consecutive inpatient admissions from 01 September 2015 to 31 August 2016. Only data of those admitted as 'inpatients' were included, as these patients would be at greatest risk of reduced mobility. Patient data were excluded for those whose anticoagulant use was contraindicated or not described by SASTH guidelines. These included patients younger than 18 years, those with hepatic failure and any haemorrhagic linked condition, those admitted with traumatic brain injury or patients receiving therapeutic anticoagulation $(n=69367$; Figure 1).

All included patient billing data were compared to the 'Venous Thromboembolism: Prophylactic and Therapeutic Practice Guideline' of the SASTH (Figure 2), and compliancy to this guideline was captured..$^{25}$

\section{Statistical analysis}

The Statistical Analysis System ${ }^{\circledR}$, SAS 9.3 ${ }^{\circledR}$ (SAS Institute Inc., USA), was used to analyse the data. Categorical variables were reported as frequencies and percentages. For this proposed study, the Pearson's chi-square test was used to determine the association between SASTH guideline compliance and clinical speciality. Cohen's $d$ value was used to determine the practical significance of the results (with $d \geq 0.1$ defined as an effect with practical significance) ${ }^{27}$

\section{Ethical consideration}

The Health Research Ethics Committee (HREC) of the Faculty of Health Sciences of the North-West University granted ethical approval, ethics number: NWU-00080-17-A1. Written permission was obtained from the study hospital group's Ethics in Research Committee.

\section{Results}

There were 373020 inpatient records that met the inclusion/ exclusion criteria for the study period. The mean age of the study population was 49.08 years (SD, 17.96 years) with a $38 \%$ to $62 \%$ male-to-female split. Patients were grouped together if they had undergone risk rating measures and further divided between those who would not require prophylaxis (low VTE risk) and those who would (medium or high VTE risk). Risk ratings were not available for $40.25 \%$ ( $n=150160)$ of the study population and contrasts with SASTH guidelines as the individualisation of prophylaxis according to VTE risk rating is recommended. Only $38.36 \%$ ( $n=85486)$ of all risk-rated patients in this data set received guideline-appropriate VTE prophylactic measures. 


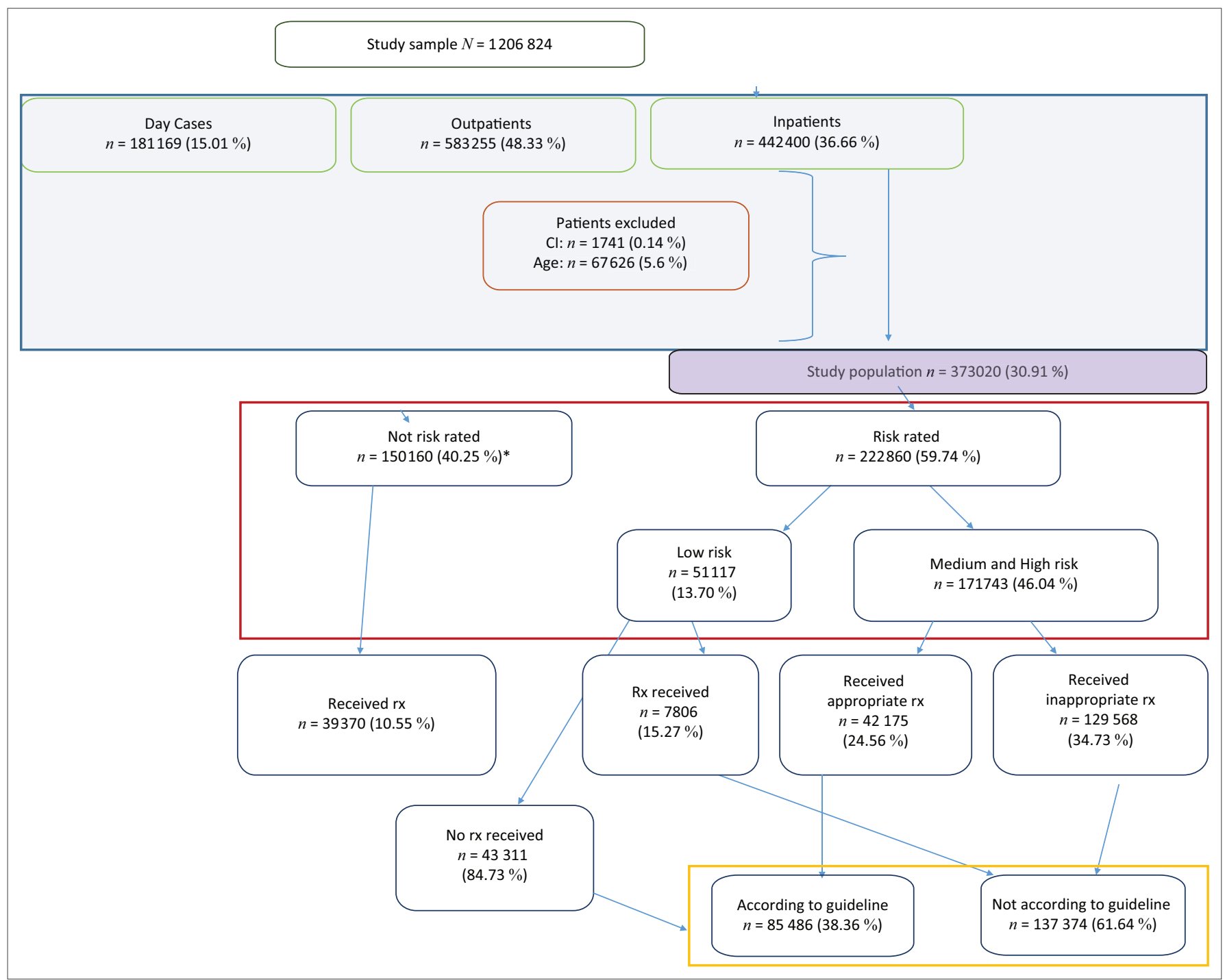

FIGURE 1: Data for study population.

Of those risk rated, $13.70 \%(n=51$ 117) were reported as having a low VTE development risk. Although guidelines do not recommend chemoprophylaxis for such patients, a total of $15.27 \%(n=7806)$ did receive some form of medicinal intervention. For those rated as medium-to-high VTE risk (prophylaxis indicated), only $24.56 \%(n=42175)$ received guideline approved prophylaxis. This may point to a disconnect between VTE rating outcomes versus clinician diagnosis.

A data summary for patients who received prophylaxis, regardless of being risk rated, was added (Table 2) in an attempt to compare characteristics for patients in whom clinicians deemed prophylaxis as appropriate. Type of prophylaxis prescribed remained consistent amongst these patients. Significantly younger patients (41.10 years) were rated as low risk compared to those at moderate to higher risk. The top three ICD10 admission codes for those rated as low DVT risk (when compared to the SASTH guidelines), however, place these 'low-risk' patients at either a medium or a high risk for VTE development. The accuracy of VTE risk ratings performed by admitting nursing staff remains in question.

Characteristics for patients who received guidelineappropriate prophylaxis are set out in Table 3. Admission codes were found to be highest for 'maternal care because of uterine scar from previous surgery' (5.01\%), and 'unspecified viral hepatitis without hepatic coma' $(1.49 \%)$ was found to be the second most prevalent. Risk ratings in this subset of patient data favoured that of a high risk for VTE development $(82.90 \%)$. Results revealed that high-risk-rated patients most often received enoxaparin or fondaparinux as prophylaxis.

It was found that enoxaparin took the highest share of prescribing chemoprophylactics by comparing the different molecules used as chemoprophylactic preventives (Table 2). Dosages of $40 \mathrm{mg}$ subcutaneous every $24 \mathrm{~h}$ were mostly billed. Fondaparinux was the second highest prescribed agent at $2.5 \mathrm{mg}$ subcutaneous every $24 \mathrm{~h}$. Unfractionated heparin (UFH; which had a higher use in those compliant with the SASTH guidelines; Table 3) was used at a dosage of 


\begin{tabular}{|c|c|c|}
\hline DVT low risk & DVT medium risk & DVT high risk \\
\hline $\begin{array}{l}\text { Associated risk factors } \\
\text { - Surgical procedures < } 30 \mathrm{~min} \\
\text { - Minor soft-tissue trauma } \\
\text { - Acute illnesses without immobility } \\
\text { - Surgical device placement without } \\
\text { immobility (i.e. CVP lines) } \\
\text { - Pregnancy and postpartum time frame } \\
\text { - Varicose veins } \\
\text { - Nephrotic syndrome }\end{array}$ & $\begin{array}{l}\text { Associated risk factors } \\
\text { - Surgical procedures }>30 \text { min } \\
\text { - Surgical/arthroscopic procedures of lower limbs } \\
\text { - Acute illnesses with immobility } \\
\text { - Acute decompensated COPD } \\
\text { - Chronic cardiac insufficiency } \\
\text { - Obesity (BMI > } 30 \mathrm{~kg} / \mathrm{m}^{2} \text { ) } \\
\text { - Oestrogen replacement therapy } \\
\text { - Elderly (> } 60 \text { years) } \\
\text { - Low-risk procedures with added patient risk }\end{array}$ & $\begin{array}{l}\text { Associated risk factors } \\
\text { - Major surgical or orthopaedic procedures: spine, cardiothoracic, } \\
\text { pelvic, hip or knee replacement or lower limbs } \\
\text { - Paralysis or stroke with paralysis } \\
\text { - Acute decompensated COPD requiring ventilation } \\
\text { - Admission to ICU } \\
\text { - Prior history of VTE/DVT } \\
\text { - Cancer or malignancies } \\
\text { - Familial thrombophilia } \\
\text { - Autoimmune/inflammatory diseases } \\
\text { - HIV/AIDS } \\
\text { - Major surgical procedures with additional patient-related risk factors } \\
\text { - Medication (i.e. tuberculostatic or thalidomide) }\end{array}$ \\
\hline $\begin{array}{l}\text { Recommended prophylaxis } \\
\text { - Ensure early mobilisation } \\
\text { - No prophylaxis recommended }\end{array}$ & $\begin{array}{l}\text { Recommended prophylaxis } \\
\text { - Enoxaparin } 40 \mathrm{mg} \text { ( } 4000 \text { anti-Xa units) s.c. daily } \\
\text { ALTERNATIVELY } \\
\text { - Dalteparin } 0.2 \mathrm{~mL} \text { ( } 2500 \text { anti-Xa units) s.c. daily } \\
\text { OR } \\
\text { - Nadroparin: } 0.3 \mathrm{~mL} \text { ( } 2850 \text { anti-Xa units) s.c. } 2 \mathrm{~h} \\
\text { pre-operatively and } 8 \mathrm{~h} \text { after surgery, followed by } 0.3 \mathrm{~mL} \\
\text { daily for } 7 \text { days for abdominal surgery } \\
\text { - Knee and hip arthroplasty: } 38 \text { anti-Xa units } / \mathrm{kg} \text { s.c. } 12 \mathrm{~h} \\
\text { pre-operatively and repeated } 12 \mathrm{~h} \text { post-operatively, } \\
\text { continue daily on Days } 1-3 \text {, with } 57 \text { anti-Xa units } / \mathrm{kg} \text { s.c. } \\
\text { from Day } 4 \text { for a minimum of } 10 \text { days. } \\
\text { - For increased bleeding risk, use IPC devices }\end{array}$ & $\begin{array}{l}\text { Recommended prophylaxis } \\
\text { - Enoxaparin } 40 \mathrm{mg} \text { ( } 4000 \text { anti-Xa units) s.c. daily } \\
\text { ALTERNATIVELY } \\
\text { - Dalteparin } 0.4 \mathrm{~mL} \text { ( } 5000 \text { anti-Xa) units s.c. daily } \\
\text { OR } \\
\text { - Nadroparin: } 0.3 \mathrm{~mL} \text { ( } 2850 \text { anti-Xa units) s.c. } 2 \mathrm{~h} \text { pre-operatively and } \\
8 \mathrm{~h} \text { after surgery, followed by } 0.3 \mathrm{~mL} \text { daily for } 7 \text { days for abdominal } \\
\text { surgery } \\
\text { - Knee and hip arthroplasty: } 38 \text { anti-Xa units/kg s.c. } 12 \mathrm{~h} \text { pre- } \\
\text { operatively and } 12 \mathrm{~h} \text { post-operatively daily on days } 1-3 \text {, then } 57 \\
\text { anti-Xa units/kg s.c. from Days } 4 \text { to } 10 \text {. } \\
\text { OR } \\
\text { - Dabigatran (only registered in hip/knee arthroplasty) } \\
\text { - } 110 \mathrm{mg} \text { pre-operatively } 4 \mathrm{~h} \text { post-operatively and } 220 \mathrm{mg} \text { daily } \\
\text { thereafter } \\
\text { For (1) creatinine clearance } 30-50 \mathrm{~mL} / \mathrm{min} \text { ), ( } 2 \text { ) patients on amiodarone } \\
\text { and ( } 3 \text { ) the elderly: } \\
\text { - } 75 \mathrm{mg} \text { pre-operatively } 4 \mathrm{~h} \text { post-operatively and } 150 \mathrm{mg} \text { daily } \\
\text { thereafter in special populations including patients with moderate } \\
\text { renal impairment } \\
\text { ALTERNATIVELY } \\
\text { - Rivaroxaban } \\
\text { - } 10 \mathrm{mg} \text { daily starting } 6 \mathrm{~h} \text { after surgery } \\
\text { OR } \\
\text { - Fondaparinux with added IPC } \\
\text { - } 2.5 \mathrm{mg} \text { s.C. daily post-operatively (only registered for prophylaxis in } \\
\text { total hip and knee arthroplasty) }\end{array}$ \\
\hline
\end{tabular}

Source: Jacobson BF, Louw S, Buller H, et al. Venous thromboembolism: Prophylactic and therapeutic practice guideline. SAMJ. 2013;103(4):260-267

COPD, chronic obstructive pulmonary disease; CVP, central venous pressure; DVT, deep vein thrombosis; ICU, intensive care unit; IPC, intermittent pneumatic compression; s.c., subcutaneous; VTE, venous thromboembolism

Figure 2: South African Society of Thrombosis and Haemostasis prophylactic practice guidelines for venous thromboembolism.

TABLE 2: Patient characteristics in those receiving prophylaxis.

\begin{tabular}{|c|c|c|c|}
\hline Patient demographics & Not rated $(n=39370)$ & Low risk rated $(n=7806)$ & Medium and high risk rated $(n=56347)$ \\
\hline Median age in years (IQR) & $54.22(S D=17.94)$ & $41.10(S D=14.38)$ & $54.9(S D=17.8)$ \\
\hline Gender - Female & $60.80 \%(n=23931)$ & $67.40 \%(n=5261)$ & $61.30 \%(n=34558)$ \\
\hline Gender - Male & $39.20 \%(n=15439)$ & $32.60 \%(n=2544)$ & $38.70 \%(n=21788)$ \\
\hline Gender - Unknown & $0.00 \%(n=0)$ & $0.00 \%(n=1)$ & $0.00 \%(n=1)$ \\
\hline \multirow[t]{2}{*}{ Primary admission } & Maternal care due to uterine scar, $4.30 \%(n=1685)$ & Maternal care due to uterine scar, $5.7 \%(n=444)$ & Maternal care due to uterine scar, $4.40 \%(n=2490)$ \\
\hline & Unstable angina, $3.8 \%(n=1483)$ & Pneumonia, unspecified, $2.7 \%(n=210)$ & Unstable angina, $3.00 \%(n=1714)$ \\
\hline \multicolumn{4}{|l|}{ Prophylaxis billed } \\
\hline \multirow[t]{3}{*}{ Anticoagulant received } & Enoxaparin, 91.80\% ( $n=36139$ doses) & Enoxaparin, 86.2\% ( $n=19176$ doses) & Enoxaparin, 89.90\% ( $n=33447$ doses) \\
\hline & Fondaparinux, $2.6 \%$ ( $n=1014$ doses) & Fondaparinux, $2.7 \%$ ( $n=1759$ doses) & Fondaparinux, $2.60 \%$ ( $n=1703$ doses) \\
\hline & Rivaroxaban $10 \mathrm{mg}, 1.2 \%$ ( $n=457$ doses) & Rivaroxaban $10 \mathrm{mg}, 1.6 \%$ ( $n=997$ doses) & Heparin, $3.99 \%$ ( $n=2513$ doses) \\
\hline \multicolumn{4}{|l|}{ Clinician specialty } \\
\hline \multirow[t]{2}{*}{ Primary specialty } & General practitioner, $22.90 \%(n=9023)$ & $\begin{array}{l}\text { Gynaecologist and obstetrician, } 28.60 \% \\
\qquad(n=2235)\end{array}$ & $\begin{array}{l}\text { Gynaecologist and obstetrician, } 24.26 \% \\
\qquad(n=13668)\end{array}$ \\
\hline & Orthopaedic surgeon, $12.40 \%(n=4871)$ & Physician, $13.10 \%(n=1026)$ & General practitioner, $13.05 \%(n=7355)$ \\
\hline
\end{tabular}

IQR, interquartile range; SD, standard deviation. 
TABLE 3: Characteristics of patients who received South African Society of Thrombosis and Haemostasis compliant prophylaxis.

\begin{tabular}{|c|c|c|}
\hline Population prophylaxis, those compliant to SASH & $n$ & $\begin{array}{l}\text { Frequency } \\
\qquad(\%)\end{array}$ \\
\hline \multicolumn{3}{|l|}{ Primary ICD10 } \\
\hline Maternal care due to uterine scar from previous surgery & 2113 & 5.01 \\
\hline Unspecified viral hepatitis without hepatic coma & 629 & 1.49 \\
\hline Unstable angina & 629 & 1.49 \\
\hline Other primary gonarthrosis & 507 & 1.20 \\
\hline Spinal stenosis, lumbar region & 488 & 1.16 \\
\hline Delivery by elective caesarean section & 438 & 1.04 \\
\hline Adult hypertrophic pyloric stenosis & 437 & 1.04 \\
\hline Adult respiratory distress syndrome & 437 & 1.04 \\
\hline Stroke, not specified as haemorrhage & 403 & 0.96 \\
\hline Bronchitis, not specified as acute or chronic & 402 & 0.95 \\
\hline Bronchopneumonia, unspecified & 402 & 0.95 \\
\hline Other and unspecified intestinal obstruction & 393 & 0.93 \\
\hline Sepsis, unspecified & 390 & 0.92 \\
\hline Insulin-dependent diabetes mellitus & 368 & 0.87 \\
\hline Primary gonarthrosis, bilateral & 351 & 0.83 \\
\hline $\begin{array}{l}\text { Chronic obstructive pulmonary disease with acute } \\
\text { exacerbation }\end{array}$ & 350 & 0.83 \\
\hline Chronic obstructive pulmonary disease, unspecified & 350 & 0.83 \\
\hline Gluteal tendinitis, pelvic region and thigh & 325 & 0.77 \\
\hline Gonarthrosis, unspecified & 325 & 0.77 \\
\hline Excessive and frequent menstruation with irregular cycle & 302 & 0.72 \\
\hline Chronic kidney disease, stage 5 & 284 & 0.67 \\
\hline Malignant neoplasm of parotid gland & 276 & 0.65 \\
\hline Malignant neoplasm of prostate & 276 & 0.65 \\
\hline Acute renal failure, unspecified & 268 & 0.64 \\
\hline Acute respiratory failure, Type 1 (hypoxemia) & 268 & 0.64 \\
\hline Liver disorders in pregnancy, childbirth & 262 & 0.62 \\
\hline Lobar pneumonia, unspecified & 262 & 0.62 \\
\hline Lumbago with sciatica, site unspecified & 256 & 0.61 \\
\hline Lumbar and other intervertebral disc disease & 256 & 0.61 \\
\hline \multicolumn{3}{|l|}{ Risk } \\
\hline DVT HIGH & 34820 & 82.56 \\
\hline DVT MEDIUM & 7355 & 17.44 \\
\hline \multicolumn{3}{|l|}{ Gender } \\
\hline Female & 26942 & 63.88144635 \\
\hline Male & 15233 & 36.11855365 \\
\hline \multicolumn{3}{|l|}{ Mean age } \\
\hline 56.45 & - & 18.19 \\
\hline \multicolumn{3}{|l|}{ Prophylaxis } \\
\hline Enoxaparin & - & 87.24 \\
\hline Fondaparinux & - & 4.04 \\
\hline Heparin & - & 3.59 \\
\hline Rivaroxaban (10 mg) & - & 2.67 \\
\hline Fraxiparine & - & 1.20 \\
\hline Nadroparin & - & 0.27 \\
\hline Dabigatran & - & 0.27 \\
\hline Mechanical prophylaxis & - & 0.72 \\
\hline
\end{tabular}

Note: $n=42$ 175, $24.56 \%$

SASTH, South African Society of Thrombosis and Haemostasis.

$5000 \mathrm{IU}$, every $8-12 \mathrm{~h}$. A dose of $10 \mathrm{mg}$ rivaroxaban novel oral anticoagulant (NOAC) was more commonly used in orthopaedic surgery patients; however, this was not limited to its registered indication for hip and knee arthroplasty. Prophylaxis used in surgery disciplines was highest for enoxaparin followed by dalteparin (NOAC). Prescribing of prophylaxis in medically ill patients favoured enoxaparin as a once in 24-h administration, followed by dalteparin at 5000 IU subcutaneous every $24 \mathrm{~h}$. The recommended doses of
TABLE 4: Distribution of South African Society of Thrombosis and Haemostasis compliancy by doctor speciality.

\begin{tabular}{|c|c|c|c|c|c|}
\hline Speciality & SASTH category & $n$ & $\begin{array}{l}\text { Observed } \\
\text { probability }\end{array}$ & $\begin{array}{l}\text { Exact Sigma } \\
\text { (two tailed) }\end{array}$ & Cohen's $d$ \\
\hline \multirow[t]{3}{*}{ Cardiologist } & Compliant & 1963 & 0.27 & 0.000 & 0.23 \\
\hline & Non-compliant & 5345 & 0.73 & - & - \\
\hline & Total & 7308 & 1.00 & - & - \\
\hline \multirow{3}{*}{$\begin{array}{l}\text { General } \\
\text { practitioner }\end{array}$} & Non-compliant & 25651 & 0.70 & 0.000 & 0.20 \\
\hline & Compliant & 10776 & 0.30 & - & - \\
\hline & Total & 36427 & 1.00 & - & - \\
\hline \multirow{3}{*}{$\begin{array}{l}\text { Gynaecologist } \\
\text { and obstetrician }\end{array}$} & Non-compliant & 16776 & 0.63 & 0.000 & 0.13 \\
\hline & Compliant & 9695 & 0.37 & - & - \\
\hline & Total & 26471 & 1.00 & - & - \\
\hline \multirow[t]{3}{*}{ Intensivist } & Non-compliant & 1 & 0.08 & 0.003 & 0.42 \\
\hline & Compliant & 12 & 0.92 & - & - \\
\hline & Total & 13 & 1.00 & - & - \\
\hline \multirow{3}{*}{$\begin{array}{l}\text { Orthopaedic } \\
\text { surgeon }\end{array}$} & Non-compliant & 10013 & 0.65 & 0.000 & 0.15 \\
\hline & Compliant & 5282 & 0.35 & - & - \\
\hline & Total & 15295 & 1.00 & - & - \\
\hline \multirow{3}{*}{$\begin{array}{l}\text { Paediatric } \\
\text { surgeon }\end{array}$} & Non-compliant & 55 & 0.34 & 0.000 & 0.16 \\
\hline & Compliant & 106 & 0.66 & - & - \\
\hline & Total & 161 & 1.00 & - & - \\
\hline \multirow[t]{3}{*}{ Paediatrician } & Compliant & 4 & 0.10 & 0.000 & 0.40 \\
\hline & Non-compliant & 35 & 0.90 & - & - \\
\hline & Total & 39 & 1.00 & - & - \\
\hline \multirow[t]{3}{*}{ Physician } & Non-compliant & 11260 & 0.66 & 0.000 & 0.16 \\
\hline & Compliant & 5809 & 0.34 & - & - \\
\hline & Total & 17069 & 1.00 & - & - \\
\hline \multirow[t]{3}{*}{ Surgeon } & Non-compliant & 12983 & 0.70 & 0.000 & 0.20 \\
\hline & Compliant & 5464 & 0.30 & - & - \\
\hline & Total & 18447 & 1.00 & - & - \\
\hline \multirow[t]{3}{*}{ Urologist } & Non-compliant & 5751 & 0.83 & 0.000 & 0.33 \\
\hline & Compliant & 1172 & 0.17 & - & - \\
\hline & Total & 6923 & 1.00 & - & - \\
\hline
\end{tabular}

SASTH, South African Society of Thrombosis and Haemostasis.

low-molecular-weight heparin (LMWH), UFH and rivaroxaban appear to have been adhered to.

Whilst chemoprophylaxis formed the largest part of all types of prophylaxis used, mechanical prevention methods were utilised in $1.2 \%$ of patients (regardless of SASTH guidelinecompliancy measure). Graduated compression stockings were slightly more favoured $(0.66 \%)$ as the mechanical prevention method compared to the use of pneumatic compression sleeves $(0.19 \%)$.

The compliance of prophylactic prescribing by speciality varied amongst prescribers $(d>0.1$ and $0.000<p>0.003$; Table 4). Intensivists $(d=0.42, p<0.003)$, paediatricians and paediatric surgeons $(d=0.2, p<0.000)$ generally were more likely to prescribe SASTH guideline-directed VTE prophylaxis (Table 4 ).

\section{Discussion}

In this single-year retrospective study, less than a quarter of patients requiring VTE prevention (those rated as medium or high VTE risk) received guideline-appropriate intervention. This contrasts with the high VTE development risk found in over $77 \%$ of patients.

Mention should also be made of the reduced percentage of RAM application found in this study. Although a risk of 
over-classification of VTE prophylactic requirement in lowrisk patients occurs with the use of a RAM, it has been proven to increase prescriber awareness of patients' VTE risk. ${ }^{3}$ This is because it has been found that prescribers do not routinely risk assess patients and base prophylactic prescribing on their perceived patient VTE development risk. ${ }^{3}$ The routine use of RAMs, on the other hand, further prevents the underdiagnoses of patients at high risk of VTE in $20 \%$ of patients, as is recommended for use in the private sector of South Africa. $^{3}$

The study population's mean age was found to be less than that reported in the TUNE-IN study but close to the mean age of 45.15 years as with the ENDORSE-Africa study. ${ }^{3,2}$ The fact that this study population contained high gynaecological admission volumes as compared to other studies may explain the age differences. Female gender distribution was found to be similar to the SA arm of the TUNE-IN Wave 2 and ENDORSE-Africa (65.6\%) studies. ${ }^{2,20}$ This reported percentage of at-risk patients is higher than the $74 \%$ average found in the TUNE-In Wave 2 study but within the range of $44 \%-80 \%$ for the ENDORSE study. ${ }^{17,20}$ The rate of appropriate prophylaxis found is much lower than the rates from both the SA TUNE-IN Wave 2 (70.9\%) and ENDORSE studies but falls in line with the ENDORSEAfrica study of guideline-compliance rates between $22 \%$ and $80 \% .{ }^{2,17,20}$ A possible reason for the lower compliance in this study is that the study population for medically ill patients included all patients older than 18 years and risk rated as exhibiting a low, medium or high thrombosis risk. The ENDORSE methodology (as followed in ENDORSEAfrica and TUNE-IN Wave 2 studies), however, only included patients older than 40 years. ${ }^{2,17,20}$ The SASTH guidelines do not specifically mention age as a predictor of risk but advocates RAMs to be used to determine VTE risk. ${ }^{25}$ This resulted in adding RAM results and ICD10 coding as inclusion criteria. Upon comparing patient age amongst those requiring prophylaxis, findings revealed that older patients were more likely to have received prophylaxis compared with those who did not receive any form of prophylactic anticoagulation. A possible explanation for this finding is that $1.4 \%$ of patients, contained in the grouping which did not receive preventative measures, was admitted with 'spontaneous vertex delivery'. These patients theoretically are assumed to be of a younger age and here the use of prenatal anticoagulation is off-label. The SASTH guidelines do recommend LMWH use prenatally in those where the benefit outweighs the risk; however, this practice requires much caution. ${ }^{25}$

This study further found that in gynaecological surgery (such as in the case of the study population's highest admission portion), the use of enoxaparin $40 \mathrm{mg}$ subcutaneous daily, starting between 6 and $8 \mathrm{~h}$ post-procedure, was prescribed. This is in compliance with the SASTH guidelines. The SASTH guidelines suggest the use of dalteparin for patients undergoing high-risk surgical procedures and is also registered as prophylaxis in patients at moderate risk of thrombosis (those undergoing abdominal and gynaecological surgery), as well as those presenting with a high risk of thrombosis (those undergoing orthopaedic surgery or suffering from an underlying malignancy). Study results reveal dalteparin use to be the second highest chemoprophylaxis molecule used in surgical procedures, which is in line with the guidelines. The high overall use of enoxaparin $(89.90 \%)$ is very similar to the TUNE-IN Wave 2 study (92\%) and is in line with SASTH recommendations where LMWH is stipulated as being superior to UFH for most prophylactic indications. ${ }^{25,28}$ One possible reason for the low use of mechanical prophylaxis in our current study population may be because of its recommended use in those at high risk of bleeding as well as the lower percentage of surgery-related admissions (excluding gynaecological surgery). Mechanical prophylaxis (pneumatic stockings) was found to be used in less than $3 \%$ of patients in the TUNE-IN Wave 2 study which is, however, higher than our study $(1.2 \%) .{ }^{28}$

Whilst practices amongst specialities differed from published guidelines, no comment can be made on the appropriateness of the prophylaxis used. This is because no clinical outcome data were available or included in the study for patients at risk of thrombosis development. One possible reason for the variance in prescriber prophylaxis may be because of the fact that the last review of the SASTH guidelines occurred in 2013 and newer anticoagulants have since been registered in South Africa. Another factor may be patients' bleeding risk (which may not have been ICD10 coded), as well as the risk of clinician litigation because of patient haemorrhage. The accuracy of VTE risk ratings may also play a role in prescribers deviating from the guidelines. It must be noted that this study's aim was not to critique prescribing practices but to illustrate occurring prophylaxis patterns as well as patient VTE risk rating spread.

Results for this study reveal that younger, female gynaecology patients make out the bulk of those who are at risk of VTE development. With such patient demographics existing in the private sector and the fact that clinicians are relying more on their own diagnosis than the results achieved from RAMs, a possibility exists that private hospital patients may require more prophylaxis than that traditionally prescribed.

\section{Limitations of this study}

The study data set is similar to the data utilised by medical aids to determine prescribers and hospital disease risk profiling. As this was a retrospective study dependent on claims information with linked pharmacy billing data, a potential exists for ICD10 case ascertainment and capturing errors, as well as under-coding. Nevertheless, because of the availability of pharmacy data, a more detailed data set was available, which enabled prescription-detail inclusion. A combination of inaccurate billing and VTE risk 
assessments may have contributed to the smaller percentage of patients found to have received prophylaxis in accordance with the SASTH guidelines. Primary ICD10 coding was used to select the study population - this may have inadvertently led to certain patients in whom anticoagulation was contraindicated, being included in the study.

\section{Conclusion}

This study confirms that most prescriber specialities do not prescribe VTE prophylaxis according to published local, consensus-derived guidelines. It can further be concluded that patients are more likely to receive some form of VTE prophylaxis if they are at high risk of VTE development. This is similar to other study findings where patients at medium risk of VTE development did not receive the required VTE prophylaxis as stipulated in the guidelines. Differences in prophylactic patterns were found between clinician specialities; however, the clinical implication of this effect remains unknown. The application of RAMs was found to have been used in less than half of the admitted patients, possibly pointing to the fact that prophylactic prescribing is more reliant on clinician diagnosis than on risk assessment. This is in contrast to the guidelines suggesting that all admitted patients should be subjected to a RAM, together with a clinician review. The concern of bleeding risk in patients, and the costs associated with VTE prophylactic under-prescribing, should not be disregarded, therefore pointing to the importance of RAM outcomes to ensure only those at risk of thrombosis are treated. Possible future research to establish reasons for clinicians not utilising RAM rating outcomes in prescribing decisions may pave the way for an improved RAM utilisation, which, in turn, may standardise VTE prophylaxis to improve outcomes.

Further review of VTE prophylaxis guidelines to include newly registered anticoagulants, as well as methods to improve the completion and acceptance of RAMs may balance the current under- and overprescribing amongst the different VTE risk categories.

\section{Acknowledgement}

The authors thank and appreciate Ms M Cockeran, MUSA, Faculty of Health Sciences, NWU, for her support with statistical computation and the study hospital group for allowing the use of the data set for the purpose of this study.

\section{Competing interests}

The authors have declared that no competing interest exists.

\section{Authors' contributions}

All authors contributed equally to this work.

\section{Funding information}

This research received no specific grant from any funding agency in the public, commercial, or not-for-profit sectors.

\section{Data availability statement}

Available data were analysed and discussed for this article.

\section{Disclaimer}

The views and opinions expressed in this article are those of the authors and do not necessarily reflect the official policy or position of any affiliated agency of the authors.

\section{References}

1. Rothwell PM, Coull AJ, Giles MF, et al. Change in stroke incidence, mortality, casefatality, severity, and risk factors in Oxfordshire, UK from 1981 to 2004 (Oxford Vascular Study). Lancet. 2004;363(9425):1925-1933. https://doi.org/10.1016/ S0140-6736(04)16405-2

2. Kingue S, Bakilo L, Mvuala R, et al. Epidemiological African day for evaluation of patients at risk of venous thrombosis in acute hospital care settings. Cardiovasc Afr. 2014;5(4):159-164. https://doi.org/10.5830/CVJA-2014-025

3. Riback WJ, Wessels PF. DVT prophylaxis in relatioN to patiEnt risk profiling - TUNEIn study. SAMJ. 2012;102(2):85-89.

4. Statistics South Africa. Mortality and causes of death in South Africa, 2016: Findings from death notification [homepage on the Internet]. c2016 [cited 2019 Mar 15] Available from: http://www.statssa.gov.za/publications/P03093/P030932016.pdf

5. Kooiman J, Van Hagen N, Iglesias Del Sol A, et al. The HAS-BLED Score identifies patients with acute venous thromboembolism at high risk of major bleeding complications during the first six months of anticoagulant treatment. PLoS One. c2015;10(4):e0122520. https://doi.org/10.1371/journal.pone.0122520

6. Luciani $\mathrm{A}$, Clement $\mathrm{O}$, Halimi $\mathrm{P}$, et al. Catheter-related upper extremity deep venous thrombosis in cancer patients: A prospective study based on Doppler US. Radiology.2001;220(3):655-660.https://doi.org/10.1177/0115426502017005324

7. Ruppert A, Steinle T, Lees M. Economic burden of venous thromboembolism: A systematic review. J Med Econ. 2011;14(1):65-74. https://doi.org/10.3111/13696 998.2010.546465

8. Fernandez MM, Hogue S, Preblick R, Kwong WJ. Review of the cost of venous thromboembolism. Clinicoecon Outcomes Res. c2015;7(1):451-462. https://doi. org/10.2147/CEOR.S85635

9. Dasta JF, Pilon D, Mody SH, et al. Daily hospitalization costs in patients with deep vein thrombosis or pulmonary embolism treated with anticoagulant therapy. Thromb Res. c2015;135(2):303-310. https://doi.org/10.1016/j.thromres.2014. 11.024

10. Grosse SD, Nelson RE, Kwame A, et al. The economic burden of incident venous thromboembolism in the United States: A review of estimated attributable healthcare costs. Thromb Res. 2016;137(1):3-10. https://doi.org/10.1016/j. thromres.2015.11.033

11. Heit JA, Spencer FA, White RH. The epidemiology of venous thromboembolism. J Thromb Thrombolysis. c2016;41(1):3-14. https://doi.org/10.1007/s11239-015-1311-6

12. Kearon C, AkI EA, Comerota AJ, et al. Antithrombotic therapy for VTE disease: Antithrombotic therapy and prevention of thrombosis, 9th ed: American College
of chest physicians evidence-based clinical practice guidelines. Chest. 2012; of chest physicians evidence-based clinical practice guidelines.
141 (2 Suppl):e419S-e494S. https://doi.org/10.1378/chest.11-2301

13. Caprini JA, Arcelus JI, Reyna JJ. Effective risk stratification of surgical and nonsurgical patients for venous thromboembolic disease. Semin Hematol. 2001;38(Suppl 5):12-19. https://doi.org/10.1016/S0037-1963(01)90094-0

14. Wilson RM, Michel P, Olsen $S$, et al. Patient safety in developing countries: retrospective estimation of scale and nature of harm to patients in hospital. BMJ. 2012;344(1):e832. https://doi.org/10.1136/bmj.e832

15. Jha AK, Larizgoitia I, Audera-Lopez C, et al. The global burden of unsafe medical care: Analytic modelling of observational studies. BMJ Qual Saf. c2013;22(10): 809-815. https://doi.org/10.1136/bmjqs-2012-001748

16. Bergmann JF, Cohen AT, Tapson VF, et al. Venous thromboembolism risk and prophylaxis in hospitalised medically ill patients. The ENDORSE global survey. Thromb Haemost. c2010;103(4):736-48. https://doi.org/10.1160/TH09-09-0667

17. Cohen AT, Tapson VF, Bergmann JF, et al. Venous thromboembolism risk and prophylaxis in the acute hospital care setting (ENDORSE study): A multinational cross-sectional study. Lancet. 2008;371(9610):387-394. https://doi.org/10.1016/ S0140-6736(08)60202-0

18. Schleyer AM, Schreuder A, Jarman KM, Logerfo JP. Adherence to guidelinedirected venous thromboembolism prophylaxis among medical and surgical inpatients at 33 Academic Medical Centers in the United States. Am J Med Qual. c2011;26(3):174-180. https://doi.org/10.1177/1062860610382289

19. Maynard G. Preventing hospital-associated venous thromboembolism: A guide for effective quality improvement. 2nd ed. Rockville: Agency for Healthcare Research and Quality; 2016 
20. Henke PK, Pannucci CJ. Venous thromboembolism risk factor assessment and prophylaxis. Phlebol. 2010;25(5):219-223. https://doi.org/10.1258/phleb.2010. 010018

21. Guyatt GH, Norris SL, Schulman S, et al. Methodology for the development of antithrombotic therapy and prevention of thrombosis guidelines: Antithrombotic therapy and prevention of thrombosis, 9th ed: American College of chest physicians evidence-based clinical practice guidelines. Chest. 2012;141(2 Suppl):53S-70S. https://doi.org/10.1378/chest.11-2288

22. Grant PJ, Greene MT, Chopra V, et al. Assessing the Caprini score for risk assessment of venous thromboembolism in hospitalized medical patients. Am J Med. 2016;129(5):528-535. https://doi.org/10.1016/j.amjmed.2015.10.027

23. Obi AT, Pannucci CJ, Nackashi A, et al. Validation of the Caprini venous thromboembolism risk assessment model in critically ill surgical patients. JAMA Surg. 2015;150(10):941-948. https://doi.org/10.1001/jamasurg.2015.1841
24. Pannucci CJ, Bailey MD, Dreszer G, et al. Validation of the Caprini risk assessment model in plastic and reconstructive surgery patients. J Am Coll Surg. 2011;212(1):105-112. https://doi.org/10.1016/j.jamcollsurg.2010.08.018

25. Jacobson BF, Louw S, Buller $\mathrm{H}$, et al. Venous thromboembolism: Prophylactic and therapeutic practice guideline. SAMJ. 2013;103(4):260-267.

26. Bilgi K, Muthusamy A, Subair M, et al. Assessing the risk for development of venous thromboembolism (VTE) in surgical patients using adapted Caprin scoring system. Int J Surg. 2016;30(1):68-73. https://doi.org/10.1016/j. ijsu.2016.04.030

27. Cohen J. Statistical power analysis for the behavioural sciences. 2nd ed. Hillsdale: Lawrence Earlbaum Associates; 1988

28. Jacobson BF, Louw S, Riback WJ. The use of VTE prophylaxis in relatioN to patiEnt risk profiling (TUNE-IN) wave 2 study. SAMJ. 2014;104(2):880-884. https://doi. org/10.7196/samj.8456 